\title{
Utilização da espessura do músculo adutor do polegar na avaliação nutricional
}

\author{
The use of the thumb adductor muscle thickness in nutritional assessment \\ Caroline Aguiar Pereira ${ }^{1}$, Juliana Gonçalves Moreno ${ }^{1}$, Raquel Milani El Kik ${ }^{2}$
}

RESUMO

Introdução: A avaliação da espessura do músculo adutor do polegar (EMAP) tem sido uma técnica descrita que surge como uma alternativa não invasiva, rápida e de baixo custo na avaliação nutricional.

Objetivo: O presente artigo tem como objetivo revisar sobre as evidências acerca da utilização da EMAP na avaliação nutricional de adultos e idosos.

Materiais e Métodos: Foi realizada uma revisão integrativa da literatura utilizando as bases de dados Medline/Pubmed, LILACS e Embase. Foram selecionados artigos publicados nos últimos 10 anos, em português e inglês.

Resultados: Foram identificados 22 artigos, dos quais 10 atenderam aos critérios de inclusão/exclusão do estudo. Os artigos analisados foram organizados nas categorias: indivíduos saudáveis, idosos institucionalizados e pacientes com condições clínicas ou cirúrgicas.

Conclusão: A EMAP pode ser utilizada na avaliação nutricional de indivíduos saudáveis e em diversas situações clínicas, apesar de ainda não existirem pontos de corte definidos para todas as populações. Para um diagnóstico nutricional mais acurado, é indispensável aliar a medida da EMAP com outros métodos e parâmetros de avaliação nutricional.

Palavras-chave: avaliação nutricional; desnutrição; antropometria; composição corporal; estado nutricional.

\begin{abstract}
Introduction: The evaluation of the adductor pollicis muscle (APM) thickness has been described as a technique which appears as non-invasive, fast and low cost alternative to nutritional assessment.

Objective: This article aims to review on the evidences concerning the use of the APM thickness standards to nutritional assessment of adults and the elderly.

Materials and Methods: An integrative literature review was conducted using Medline/Pubmed, LILACS and Embase. We have selected articles published in the last 10 years, in Portuguese and English.

Results: Twenty two articles were identified, of which 10 met the criteria for inclusion/exclusion from the study. The analyzed articles were organized in the categories: healthy subjects, institutionalized elderly and medical or surgical patients.

Conclusions: The APM standard can be used in the nutritional assessment of healthy subjects and in various clinical situations, although there are not cutoff points for all populations. For a more accurate nutritional diagnosis, it is essential to combine the APM standard with other methods and parameters of nutritional assessment.
\end{abstract}

Keywords: nutrition assessment; malnutrition; anthropometry; body composition; nutritional status.

${ }^{1}$ Nutricionista graduada pela Faculdade de Enfermagem, Nutrição e Fisioterapia da Pontifícia Universidade Católica do Rio grande do Sul (PUCRS). Pós-graduanda em Nutrição Clínica e Doenças Crônicas pelo Instituto de Educação e Pesquisa do Hospital Moinhos de Vento.

${ }^{2}$ Nutricionista. Doutoranda em Gerontologia Biomédica pela PUCRS. Professora Adjunta do Curso de Nutrição da FAENFI (PUCRS). 


\section{INTRODUÇÃo}

A avaliação nutricional (AN) é um processo que visa identificar problemas relacionados à nutrição ${ }^{1} \mathrm{e}$ compreende vários parâmetros ${ }^{2}$. Deve ser realizada com cautela, pois todas as medidas utilizadas podem ser influenciadas pela presença de doenças ou trauma e não há um método sem pelo menos uma limitação" Alterações fisiológicas, mudanças na composição corporal próprias da idade e outras doenças associadas também dificultam a AN do idoso ${ }^{3}$.

$\mathrm{Na}$ história clínica, vários fatores podem diminuir a eficiência na obtenção das informações ${ }^{4}$. $\mathrm{Na}$ antropometria, alterações decorrentes de certas patologias também podem levar a uma menor precisão e comprometer a determinação de um diagnóstico acurado $^{5}$. Na avaliação de exames laboratoriais, é essencial uma interpretação cautelosa, pois podem ser influenciados por enfermidades, uso de drogas ou estresse. O mesmo ocorre com o exame físico, que requer a atenção e o conhecimento da história clínica do paciente $^{6}$. O exame físico com ênfase na avaliação muscular deve ser valorizado para avaliar a reserva protéica muscular ${ }^{7}$. A avaliação muscular subjetiva é orientada para quatro grupos musculares envolvidos nas atividades rotineiras diárias e inclui o músculo adutor do polegar 8 .

A avaliação da espessura do músculo adutor do polegar (EMAP) é uma técnica que surgiu como uma alternativa não invasiva, rápida e de baixo custo para a AN. O músculo adutor do polegar é responsável pela força de pinçamento do polegar ${ }^{9}$ e, por ser plano e estar fixado entre duas estruturas ósseas, é o único músculo do corpo humano que permite a medida direta de sua espessura com o paquímetro de Lange ${ }^{10}$. Em 2004, Lameu et al. ${ }^{9}$ realizaram um dos primeiros estudos para avaliar a EMAP com a finalidade de utilizar esta medida como um parâmetro antropométrico.

Oliveira et al. ${ }^{11}$, em 2010, compararam a efetividade de diferentes métodos utilizados na $\mathrm{AN}$ de pacientes adultos e idosos ambulatoriais que sofreram acidente vascular encefálico. Neste estudo os resultados apontam para a aplicabilidade da EMAP na prática clínica como método de AN para essa população, especialmente em idosos, sendo importante na identificação precoce do risco nutricional.

Andrade et al. ${ }^{12}$, em 2005, realizaram um estudo com 99 pacientes hospitalizados portadores de doença cardíaca com indicação cirúrgica e avaliaram a EMAP no pré-operatório. A EMAP apresentou uma forte tendência de correlação com o tempo de internação hospitalar e somente nos pacientes com importante perda do trofismo do músculo adutor houve uma associação significativa com a evolução pós-operatória para complicações infecciosas. Nenhum parâmetro isolado de AN mostrou-se superior e eficiente como índice prognóstico único adequado para morbidez séptica e não séptica, mortalidade e tempo de internação hospitalar.

Entretanto, em 2010, Freitas et al. ${ }^{13}$ realizaram um estudo com 82 pacientes adultos e idosos, oncológicos e hospitalizados e a EMAP não se revelou um bom indicador prognóstico antropométrico, possivelmente pela presença de um menor comprometimento do estado nutricional observada na maioria do pacientes e por permanecerem menos tempo no ambiente hospitalar.

Outro estudo realizado por Cobêro et al. ${ }^{14}$, em 2012, com 112 pacientes, com doenças cardiovasculares hospitalizados, também não identificou correlação positiva entre a EMAP e os demais parâmetros da AN. Não foi possível determinar um ponto de corte para a classificação do estado nutricional, tendo em vista a grande proporção de sobrepeso e obesidade na população estudada. Sendo assim, o objetivo deste trabalho foi revisar sobre as evidências acerca da utilização da EMAP na AN.

\section{MATERIAIS E MÉTODOS}

Foi realizada uma revisão integrativa (RI) da literatura ${ }^{15,16}$, entre março e novembro de 2013, seguindo as seis etapas preconizadas: identificação do tema e seleção da questão de pesquisa; estabelecimento de critérios de inclusão e exclusão; definição das informações a serem extraídas; avaliação dos estudos incluídos na Rl; interpretação dos resultados; e apresentação da revisão/síntese do conhecimento $^{15}$

O tema desta RI foi a utilização da EMAP na AN e 
busca responder a questão norteadora: Quais as evidências disponíveis na literatura acerca da utilização da EMAP na AN?

Foi realizada a busca de artigos publicados no período de 2003 a 2013 nas bases de dados Medline/Pubmed, LILACS e Embase. Com apoio na questão norteadora utilizaram-se os termos indexados: avaliação nutricional, desnutrição, antropometria, composição corporal, estado nutricional, músculo adutor do polegar. Para os cruzamentos adotou-se a seguinte estratégia de busca: "músculo adutor do polegar" and "composição corporal" or "antropometria" or "desnutrição" or "avaliação nutricional”. Os equivalentes também foram utilizados no idioma inglês, através das bases de dados Medline/Pubmed e Embase. Foram considerados critérios de inclusão: artigos originais publicados nos idiomas português, espanhol e inglês, que se enquadraram na questão da pesquisa.

Para a análise dos artigos foi utilizado um instrumento com a seguinte estrutura: autores do artigo e ano da publicação, método e principais achados do estudo. Este estudo segue os princípios éticos preconizados para pesquisas desta natureza, respeitando a autoria e os dados apresentados nos artigos.

\section{RESULTADOS E DISCUSSÃO}

Foram localizados vinte e dois artigos nas bases de dados pesquisadas relacionados com a EMAP. Deste total, doze artigos foram selecionados na base Medline/Pubmed, sendo que três foram eliminados por não se enquadrarem à questão norteadora da pesquisa, resultando em nove artigos selecionados. $\mathrm{Na}$ base de dados Lilacs, foram identificados dez artigos, sendo que nove foram excluídos da análise, por já terem sido utilizados ou por não responderem à questão norteadora. $\mathrm{Na}$ base de dados Embase, foram encontrados nove artigos, os mesmos já identificados na base de dados Medline/Pubmed. Assim, a revisão foi realizada com dez artigos que se adequaram aos critérios de inclusão estabelecidos. Dos artigos incluídos, todos foram escritos nos últimos 10 anos, a maioria entre 2004 e 2012. O Brasil teve o maior número de publicações, com nove trabalhos, e os Estados Unidos teve apenas um trabalho.
Quanto ao delineamento dos artigos avaliados, evidenciou-se: estudos transversais, estudos de coorte, estudos prospectivos e um ensaio clínico randomizado. Os artigos analisados foram identificados como Estudos sobre a utilização da EMAP na AN - nas seguintes categorias: 1. Indivíduos Saudáveis; 2. Idosos Institucionalizados; 3. Pacientes com condições clínicas ou cirúrgicas. Na tabela 1 encontra-se o resumo dos estudos.

\section{Indivíduos saudáveis}

Um dos relatos mais antigos sobre a utilização da EMAP na AN foi em 2004, registrado por Lameu et al. ${ }^{9}$, onde avaliaram a EMAP de adultos saudáveis, a fim de utilizá-la como um parâmetro antropométrico. Foram incluídos 421 participantes com idade entre 18 e 87 anos de vários municípios do Rio de Janeiro. Este estudo forneceu as primeiras estimativas da EMAP em indivíduos saudáveis como um parâmetro antropométrico.

No mesmo ano, Lameu et al. ${ }^{17}$, publicaram outro estudo utilizando a mesma amostra, porém comparando a EMAP com IMC, AMB, dobra cutânea triciptal (DCT), percentual de gordura e circunferência da panturrilha (CP). A EMAP apresentou correlação positiva com as variáveis antropométricas que estimam a massa muscular, mas não se correlacionou com os parâmetros que estimam a massa de gordura. Também observaram que a utilização da EMAP na $\mathrm{AN}$ é facilmente reproduzível por observadores independentes.

Em 2008, Budziareck et al. ${ }^{18}$ realizaram um estudo com 300 adultos e idosos saudáveis, com idade entre 18 e 90 anos, com o objetivo de determinar valores de referência para a força de preensão palmar (FPP) e correlacionar com a EMAP. Concluíram que o uso combinado da FPP e da EMAP pode ser útil como um método para a AN.

Em 2009 Gonzalez et al. ${ }^{19}$ realizaram um estudo, com 300 adultos e idosos saudáveis, com a faixa etária ente 18 e 90 anos, a fim de determinar valores para a EMAP. Concluíram que os valores da EMAP variam de acordo com sexo e idade em indivíduos saudáveis e nutridos e que a aferição da EMAP exige bastante cuidado para a garantia da qualidade dos valores aferidos. 
TABELA 1 - Estudos sobre a utilização da espessura do músculo adutor do polegar na avaliação nutricional.

\begin{tabular}{|c|c|}
\hline Autor/Ano & Métodos \\
\hline Lameu et al. ${ }^{9}$ (2004) & $\begin{array}{l}\text { Ensaio clínico randomizado com } 421 \text { adultos } \\
\text { e idosos saudáveis, com a faixa etária entre } \\
\qquad 18 \text { e } 87 \text { anos. }\end{array}$ \\
\hline Lameu et al. ${ }^{17}(2004)$ & $\begin{array}{c}\text { Estudo transversal com } 421 \text { adultos e idosos } \\
\text { saudáveis, com a faixa etária entre } 18 \text { e } 87 \\
\text { anos. Comparou a EMAP com IMC, AMB, } \\
\text { DCT, percentual de gordura e CP. }\end{array}$ \\
\hline Budziareck et al. ${ }^{18}(2008)$ & $\begin{array}{l}\text { Estudo transversal com } 300 \text { adultos e idosos } \\
\text { saudáveis, com idade entre } 18 \text { e } 90 \text { anos. }\end{array}$ \\
\hline
\end{tabular}

Gonzalez et al. ${ }^{19}(2009)$

Volpini et al. ${ }^{20}(2012)$

Bragagnolo et al. ${ }^{21}$ (2009)

Caporossi et al. ${ }^{22}$ (2012)

Oliveira et al. ${ }^{23}(2012)$

Poziomyck et al. ${ }^{24}$ (2012)

da Silva et al. ${ }^{25}(2013)$
Estudo transversal realizado em 300 pessoas saudáveis, adultos e idosos entre 18 e 90 anos.

Estudo transversal realizado em uma instituição de longa permanência, com 102 idosos. Foram coletados IMC, DCT, AMB,

EMAP, FPP e exames bioquímicos.

Estudo transversal com 87 pacientes, maiores de 18 anos, candidatos à cirurgia do TGI. A AN foi feita pela ASG, antropometria, exames bioquímicos $e$ EMAP.

Estudo de coorte, com 248 pacientes a partir de 18 anos, internados para tratamento médico, cirúrgico ou intensivo. A EMAP foi correlacionada com mortalidade, tempo de ventilação mecânica e de internação.

O estudo de coorte incluiu 143 pacientes a partir de 18 anos em hemodiálise. Foram aferidos IMC, percentual de perda de peso, $\mathrm{CB}, \mathrm{AMB}, \mathrm{DCT}, \mathrm{EMAP}$, exames bioquímicos e bioimpedância.

Estudo prospectivo com 74 pacientes entre 34 e 83 anos, submetidos a ressecção de tumor do TGI superior. Foi utilizada ASG, percentual de perda de peso, IMC, CB, AMB, DCT, exames bioquímicos e EMAP

Estudo prospectivo com 43 pacientes acima de 18 anos, com câncer. Foram utilizados ASG, IMC, DCT, CB, AMB, EMAP e FPP.
Principais Achados

Fornece as primeiras estimativas da EMAP em indivíduos saudáveis como um parâmetro antropométrico.

A EMAP apresentou correlação positiva com as variáveis antropométricas que estimam a massa muscular, mas não se correlacionou com os parâmetros que estimam a massa de gordura.

A EMAP mostrou uma forte correlação com a FPP. O uso combinado da FPP e da EMAP pode ser útil como um método para a $A N$.

Os valores da EMAP variam de acordo com sexo e idade em indivíduos saudáveis e nutridos.

Houve significância estatística entre a EMAP e CB e AMB no sexo masculino. Sugere-se cautela no uso e na interpretação dessa medida, aliando sempre outros parâmetros de AN.

A EMAP é um método de fácil execução, baixo custo, confiável e transmite segurança na $A N$, podendo ser usada também em pacientes cirúrgicos.

Pacientes com desnutrição grave mostraram baixos valores para a EMAP, comparados com pacientes bem nutridos. A EMAP pode ser usada como um indicador prognóstico em pacientes criticamente doentes.

A EMAP foi bem correlacionada com IMC, $\mathrm{CB}, \mathrm{AMB}$, percentual de perda de peso, creatinina, albumina e bioimpedância. A EMAP pode ser um parâmetro útil para o diagnóstico precoce da desnutrição e um marcador de risco para mortalidade nesta população.

A EMAP e a ASG são parâmetros confiáveis de previsão de mortalidade em pacientes submetidos a ressecção de tumor do TGI superior.

Foi encontrada diferença significativa entre os valores de $\mathrm{CB}, \mathrm{AMB}$, EMAP e FPP, sugerindo que estes parâmetros podem ser úteis na diferenciação de pacientes nutridos e desnutridos. Houve pequena concordância entre os parâmetros utilizados, como a EMAP e ASG.

\section{Idosos institucionalizados}

Em 2012, Volpini et al. $^{20}$, a fim de definir o perfil nutricional de idosos institucionalizados, realizaram um estudo em uma instituição de longa permanência para idosos, com 102 moradores. Foram coletados IMC,
DCT, AMB, EMAP, FPP e exames bioquímicos. Foi identificada significância estatística entre a EMAP e a $C B$, e EMAP e AMB no grupo masculino. Esse parâmetro ainda é pouco aplicado e testado na prática de estudos populacionais e, principalmente, com idosos 
institucionalizados. Sugere-se cautela no uso e na interpretação dessa medida, recomendando sempre aliá-la a outros parâmetros

\section{Pacientes com condições clínicas ou cirúrgicas}

Bragagnolo et al. ${ }^{21}$ publicaram um estudo em 2009 com o objetivo de verificar se a utilização da EMAP é confiável na AN de 87 pacientes, maiores de 18 anos, candidatos à cirurgia de grande porte no trato gastrointestinal (TGI). Foi realizada ASG, antropometria, albumina, linfocitometria e EMAP. Todas as medidas antropométricas se correlacionaram de modo significativo com a EMAP. Concluíram que a EMAP é um método de fácil execução, baixo custo, confiável e transmite segurança na $\mathrm{AN}$, podendo ser usada também em pacientes cirúrgicos.

Caporossi et al. ${ }^{22}$, em 2012, realizaram um estudo com 248 pessoas de ambos os sexos a partir de 18 anos, internadas para tratamento médico, cirúrgico ou intensivo, para avaliar se a EMAP pode ser utilizada como indicador prognóstico. A EMAP foi correlacionada com mortalidade, tempo de ventilação mecânica e tempo de internação. Pacientes com desnutrição grave mostraram baixos valores da EMAP, comparados com os valores dos pacientes bem nutridos. $A$ análise dos resultados mostrou que a EMAP pode ser usada como indicador de prognóstico, sendo uma ferramenta valiosa para estimar o estado nutricional e prever mortalidade em pacientes críticos.

Oliveira et al. ${ }^{23}$, em 2012, recrutaram 143 pacientes em hemodiálise, a partir de 18 anos, para analisar a EMAP e investigar sua correlação com antropometria, exames bioquímicos e bioimpedância e, juntamente, associar com mortalidade e morbidade. Este é o primeiro artigo com pacientes com insuficiência renal crônica em que os valores da EMAP foram bem correlacionados com idade, sexo, comorbidades, IMC, exames bioquímicos e bioimpedância. A EMAP pode ser um parâmetro útil para o diagnóstico precoce da desnutrição e um marcador de risco para internação e mortalidade nesta população.

Poziomyck et al. ${ }^{24}$, em 2012, realizaram um trabalho com 74 pacientes entre 34 e 83 anos, submetidos a ressecção dos tumores do TGI superior a fim de determinar qual método de AN capaz de prever melhor resultado. Foi utilizada a ASG, percentual de perda de peso, IMC, CB e AMB, DCT, EMAP e exames bioquímicos. Concluíram que a EMAP e a ASG podem ser parâmetros confiáveis de previsão de mortalidade em pacientes submetidos a ressecção dos tumores do TGI superior e podem ser facilmente utilizados na prática clínica.

Da Silva et al. ${ }^{25}$, avaliaram o estado nutricional de 43 pacientes, maiores de 18 anos, durante o tratamento de câncer, sendo que a EMAP foi uma das medidas utilizadas. Foram verificados IMC, DCT, CB, AMB, e FPP dos pacientes. Houve pequena concordância entre os parâmetros utilizados como a EMAP e ASG. Foi encontrada diferença significativa entre os valores da CB, AMB, EMAP e FPP, sugerindo que estes parâmetros podem ser úteis na diferenciação de pacientes nutridos e desnutridos, se diferentes valores de corte forem adotados.

Por ser um método simples, não invasivo e de baixo custo, a utilização da EMAP vem sendo estudada na AN nas diversas condições clínicas ${ }^{9,11-14,17-25}$. Alguns autores concluem que a EMAP varia de acordo com sexo, idade e patologias da amostra avaliada e devem ser estratificados em relação a estes achados ${ }^{18,19}$.

A maioria dos estudos apresentam correlação positiva entre a utilização da EMAP com as variáveis antropométricas e bioquímicas, dando credibilidade para a utilização da mesma na AN. A EMAP pode ser um parâmetro apropriado para o diagnóstico precoce da desnutrição em algumas populações específicas, um marcador de risco para mortalidade, e um indicador prognóstico em pacientes criticamente enfermos ${ }^{21-25}$. Juntamente com outros métodos, como a FPP, a EMAP pode ser favorável para a $\mathrm{AN}^{18}$.

Como foi descrito, todos os métodos de $\mathrm{AN}$ apresentam limitações e a EMAP pode ser uma boa alternativa nos casos em que não é possível utilizar outros parâmetros de $\mathrm{AN}$, pois pode ser aferida em indivíduos saudáveis e em diversas situações clínicas, tendo seu uso restrito a pacientes que não possuem as mãos.

Ainda será necessário determinar valores ou pontos de corte da EMAP para diversas populações, patologias 
e/ou condições clinicas. Para um diagnóstico nutricional mais acurado, é indispensável aliar a medida da EMAP com outros métodos e parâmetros de AN.

\section{REFERÊNCIAS}

1. Dias MCG, Van Aanholt DPJ, Catalani LA, Rey JSF, Gonzales MC, Coppini L, Franco Filho JW, Paes-Barbosa MR, Horie L, Abrahão V, Martins C. Triagem e avaliação do estado nutricional: projeto diretrizes. Brasília (DF): Associação Médica Brasileira e Conselho Federal de Medicina; 2011.

2. Width $M$, Reinhard T. Avaliação e suporte Nutricional. In Width M, Reinhard T. Mds: manual de sobrevivência para nutrição clínica. Rio de Janeiro: Guanabara Koogan; 2010. p. 3-29.

3. Pfrimer K, Ferriolli E. Avaliação nutricional do idoso. In Vitolo MR. Nutrição da gestação ao envelhecimento. Rio de Janeiro: Rubio; 2008. p. 435-49.

4. Baxter YC, Waitzberg DL, Peres G. Métodos nãoconvencionais; estudo dietético e medida da qualidade de vida. In: Waitzberg DL. Nutrição oral, enteral e parenteral na prática clínica. $3^{\underline{a}}$ ed. São Paulo: Atheneu; 2000. p. 305-19.

5. Gibson, R. Reproducibility in dietary assessment. In: Gibson, R. Principles of nutritional assessment. $2^{\text {nd }}$. ed. New York: Oxford University Press; 1990. p. 155-61, 187205.

6. Brito S, Dreyer E. Terapia nutricional: conduta do nutricionista. Campinas: UNICAMP/ Hospital de Clínicas; 2003.

7. Grant JP. Functional and dynamics techniques for nutritional assessment. In: Handbook of Total Parenteral Nutrition. $2^{\text {nd }} . e d$. W.B. Philadelphia: Saunders Company: John Palmer Grant; 1992. p. 49.

8. Lameu EB. Avaliação Muscular Subjetiva. In: Lameu EB, Andrade PVB, Luiz RR. Clínica nutricional. São Paulo: Revinter; 2005. p.179-87.

9. Lameu EB, Gerude MF, Corrêa RC, Lima KA. Adductor policis muscle: a new anthropometric parameter. Rev Hosp Clín Fac Med. 2004;59(2):57-62

10. Lameu EB. Músculo adutor do polegar. In: Lameu EB, Gerude MF. Clínica nutricional. São Paulo: Revinter; 2005. p.189-96.

11. Oliveira DR, Frangella VS. Adductor pollicis muscle and hand grip strength: potential methods of nutritional assessment in outpatients with stroke. Einstein. 2010; 8(4 Pt 1):467-72.

12. Andrade FN, Lameu EB, Luiz RR. Musculatura Adutora do Polegar: um novo índice prognóstico em cirurgia cardíaca valvar. Rev SOCERJ. 2005;18(5):384-91.

13. Freitas BJSA, Mesquita LC, Teive NJV, Souza SR. Antropometria clássica e músculo adutor do polegar na determinação do prognóstico nutricional em pacientes oncológicos. Rev Bras Canc. 2010;56(4):415-22.
14. Cobêro FE, Gomes MCB, Silva AP, Bernardi JLD, McLellan KCP. Adductor Pollicis Muscle Measurement is Associated with Anthropometric Indicator of Muscle Mass and Fat Mass of Hospitalized Patients. Rev Soc Bras Alim Nutr. 2012;37(2):174-82.

15. Mendes KDS, Silveira RCCP, Galvão CM. Revisão Integrativa: método de pesquisa para a incorporação de evidências na saúde e na enfermagem. Texto Contexto Enferm. 2008;17(4): 758-64.

16. De Souza MT, da Silva $M D$, de Carvalho R. Revisão integrativa: o que é e como fazer. Einstein. 2010;8(1 Pt 1):102-6.

17. Lameu EB, Gerude MF, Campos AC, Luiz RR. The thickness of the adductor pollicis muscle reflects the muscle compartment and may be used as a new anthropometric parameter for nutritional assessment. Curr Opin Clin Nutr Metab Care. 2004;7(3):293-301.

18. Budziareck MB, Duarte RRP, Silva MCGB. References values and determinants for handgrip strength in healthy subjects. Clin Nutr. 2008; 27:357-62.

19. Gonzalez MC, Duarte RRP, Budziareck MB. Adductor pollicis muscle: Reference values of this thickness in a healthy population. Clin Nutr. 2010; 29:268-71.

20. Volpini MM, Frangella VS. Avaliação nutricional de idosos institucionalizados. Einstein. 2013;11(1):32-40.

21. Bragagnolo R, Caporossi FS, Nascimento DBD, Nascimento JEA, TCBC-MT. Espessura do músculo adutor do polegar: um método rápido e confiável na avaliação nutricional de pacientes cirúrgicos. Rev Col Bras Cir. 2009; 36(5):371-6.

22. Caporossi FS, Caporossi C, Nascimento DBD, Nascimento JEA. Measurement of the thickness of adductor pollicis muscle as a predictor of outcome in critically ill patients. Nutr Hosp. 2012; 27(2):490-5.

23. De Oliveira MCO, Kubrusly M, Mota RS, Choukroun G, Neto JB, Da Silva CAB. Adductor pollicis muscle thickness: a promising anthropometric parameter for patients with chronic renal failure. J Ren Nutr. 2012; 22(3):307-16

24. Poziomyck AK, Weston AC, Lameu EB, Cassol OS, Coelho LJ, Moreira LF. Preoperative nutritional assessment and prognosis in patients with foregut tumors. Nutr Cancer. 2012;64(8):1174-81.

25. Da Silva JB, Maurício SF, Bering T, Correia MITD. The relationship between nutritional status and the glasgow prognostic score in patients with cancer of the esophagus and stomach. Nutr Cancer. 2013;65(1):25-33.

\section{Endereço para correspondência}

Juliana Goncalves Moreno

Rua Miguel Tostes $n^{\circ} 225$, apto. 201

Porto Alegre/RS - CEP 90430-061

Telefone: +55 5182057335

E-mail: julianagmoreno@outlook.com 